$$
\text { DOE/T:/912846.72 }
$$

\title{
NITRATION OF POLYNUCLEAR AROMATIC HYDROCARBONS IN COAL COMBUSTORS AND EXHAUST STREAMS
}

Quarterly Report for the

Period April 1, 1992 to June 30, 1992

O 3 TI

\author{
Prepared for \\ THE UNITED STATES DEPARTMENT OF ENERGY \\ Kamalendu Das \\ Project Officer \\ Morgantown Energy Technology Center \\ P.O. Box 880; MS E02 \\ Morgantown, WV 26507
}

\begin{abstract}
Submitted by
Ms. Liya Yu, Mr. John Dadamio, Asst. Prof. Lynn Hildemann and Asst. Prof. Stephen Niska
\end{abstract}

This report was prepared as an account of work sponsored by the United States Government. Neither the United States nor the United States Department of Energy, nor any of their employees, makes any warranty, express or implied, or assumes any legal liability or responsibility for the accuracy, completeness, or usefulness of any information, apparatus, product or process disclosed, or represents that its use would not infringe privately owned rights. Reference herein to any specific commercial product, process, or service by trade name, mark, manufacturer, or otherwise, does not necessarily constitute or imply its endorsement, recommendation, or favoring by the United States Government or any agency thereof. The views and opinions of authors expressed herein do not necessarily state or reflect those of the United States Government or any agency thereof.

US/DOE Patent Clearance is not required prior to the publication of this document.

February 1993
Environmental Engineering Group
Civil Engineering Department and

High Temperature Gasdynamics Laboratory

Mechanical Engineering Department

Stanford University

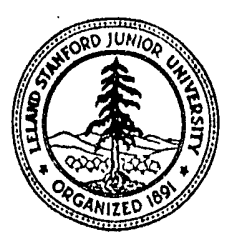






2. INTRODUCTION TO 1 HE PROGRAM............................................ 1

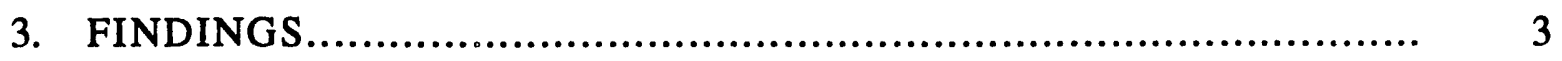

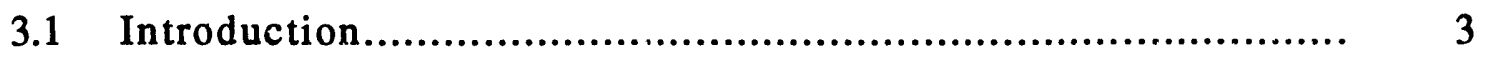

3.2 Sample Preparation............................................................... 4

3.2.1 Operating Conditions for Primary and Secondary Coal Tars...... 4

3.2.2 Experimental Results................................................... 8

3.3 Sample Analysis.............................................................. 11

3.3.1 Acquisition of Standards............................................. 11

3.3.2 Prefractionation Results............................................. 13

4. SHORT RANGE OBJECTIVES..................................................... 16 


\section{SUMMARY}

Still pending approval of our environmental impact statement and release of funds for this project, we have continued moving forward with contingency financial backing from the Mechanical and Civil Engineering Departments at Stanford. During the period April 1, 1992 to June 30,1992 both graduate students continued to become familiar with their laboratory systems.

Regarding PAH preparation, our efforts this quarter were directed at preparing PAH samples at well-controlled extents of primary devolatilization. In the long $(12.5 \mathrm{~cm})$ hot zone, preliminary studies with a Pit. \#8 hvA bituminous coal showed that a furnace temperature of 1380 $\mathrm{K}$ yields the best resolution of primary devolatilization from secondary pyrolysis. There is no soot at all in the PAH samples collected during the first half of tar evolution. Although some soot is present in the total sample of all tar from primary devolatilization, it never amounts to more than 10 $\%$ of the aerosol and is usually less than $5 \%$. Also, the maximum weight loss observed with the new operating conditions is nearly $50 \mathrm{wt} . \%$ daf, which compares favorably to our previous value of $55 \mathrm{wt} . \%$ for this coal.

Regarding PAH analysis during this quarter, the assembly of the gravity-flow column chromatographic system for prefractionating the coal tar was completed. Standard compounds spanning a wide range of polarities and molecular weights were selected and purchased. These compounds were utilized to begin testing and refining the prefractionation procedure. 


\section{INTRODUCTION TO THE PROGRAM}

Nitro-polynuclear aromatic hydrocarbons (nitro-PAH) are the predominant mutagens on respirable particles from coal-fired boilers. Since nitro-PAH are not primary products of coal devolatilization, their formation must involve secondary chemistry at elevated temperatures. However, it is not known where in the combustion or exhaust processes they form, which reaction species are involved, or how concentrations are influenced by operating conditions. Results from this study will help to relate the environmental impact of mutagenic emissions to boiler firing strategies.

The objectives of this three-year project are to (1) identify the conditions which promote the nitration of PAH during primary combustion, reburning, hot gas cleanup, and particulate removal; and (2) investigate the potential relationship between NOx abatement and PAH nitration. A novel coal flow reactor burning actual coal products operates over the domains of heating rates, temperatures, fuel-equivalence ratios, and residence times in utility boilers. A fluidized bed will be built for studies of simulated hot gas cleanup at lower temperatures. Gas chromatography with chemiluminescence detection will measure the aggregate amount of nitro groups present, to determine when nitro-PAH first appear, and how nitration is affected by the operating conditions. Tars from primary and secondary pyrolysis and oxidative pyrolysis will be fractionated into chain hydrocarbons, polynuclear aromatics, oxygenated species, and a basic fraction, so that their ring number distribution can be monitored with high performance liquid chromatography. 


\section{FINDINGS}

\subsection{Introduction}

Meeting the objective of this program involves two broad tasks: (1) Preparing the polynuclear aromatic hydrocarbons (PAH) under closely monitored pulverized fuel (p. f.) firing conditions; and (2) analyzing the PAH samples to monitor extents of nitration, ring number distributions, etc. While both activities are essential to this project, they involve scientific principles which are almost completely unrelated. So in this and all future reports, our findings will be segregated according to their relation to either sample preparation or sample analysis. In actuality, Profs. Niksa and Hildemann each have a student working on these respective parts of this project.

Regarding PAH preparation, our efforts from April through June 1992 were directed at preparing PAH at well-defined extents of primary devolatilization. The search for new operating conditions in the longer furnace (described in the second quarterly report) was concluded: the best furnace temperature is $1380 \mathrm{~K}$. Results presented below characterize the transient evolution of weight loss and tar as well as the percentage of soot in the aerosol products throughout primary devolatilization.

Regarding PAH analysis, our main efforts this quarter were aimed at examining the effects of sample size and solvent quantities on the recovery and fractionation of an organic sample, using PAH standards. Initial characterization of the prefractionation procedure using standard compounds was considered an important preliminary step to be conducted before fine-tuning the separation methodology using complex tar samples. The results presented in Section 3.3 describe the methodologies used to fractionate the standard samples while maximizing their recovery. 


\subsection{Sample Preparation}

\subsubsection{Operating Conditions for Primary and Secondary Coal Tars}

As explained in our second quarterly report, we decided to conduct studies of primary devolatilization, secondary pyrolysis, and oxidative pyrolysis in a furnace with a $12.5 \mathrm{~cm}$ hot zone. Formerly, primary devolatilization experiments were conducted in a $5 \mathrm{~cm}$ hot zone. Operating conditions can be adjusted to compensate for the longer hot zone by increasing entrainment gas flowrate, to reduce residence time; by lowering suspension loading, to lower gas and suspension temperatures; and by lowering the furnace temperatures. We knew at the beginning of this quarter that, among the three possible ways to compensate for the longer furnace tube, only lowering the furnace temperature is really effective. Coal temperatures are too insensitive to loading (in the range near $300 \mathrm{\#} / \mathrm{cm}^{3}$ ) and gas flowrates are limited by the transition to turbulence that causes deposition problems.

The viable range of operating conditions is already specified for loading, at $300 \# / \mathrm{cm}^{3}$, and for entrainment gas velocity, from 0.18 to $1 \mathrm{~m} / \mathrm{s}$. Lower velocities admit disturbances from buoyancy and higher values promote the transition to turbulence that causes deposits. Preliminary studies surveyed a range of values of furnace temperatures to pin down the final operating conditions.

Our strategy" relied on past data from primary devolatilization and secondary pyrolysis studies in this lab that show that the presence of soot is a definitive indicator for the extent of secondary pyrolysis. Soot yields are based on solubility in tetrahydrofuran (THF), and tue following scheme for product recovery and separation. 
Products are segregated into bulk solid particles, fine aerosol (when condensibles are present), and noncondensible gases in the aerodynamic classifier, called a centripeter, shown in Fig. 1. In the laboratory, the centripeter is located downstream of the furnace, beyond a quench nozzle that blasts a stream of cold argon into the process stream. Beyond the inlet nozzle to the centripeter, the process stream is split between opposing nozzles. Ninety-five percent of the flow follows the streamlines in the sketch into the annulus. Since the aerosol particles are typically a few microns in diameter, they are convected into the annulus and ultimately onto filters. Particles with sufficient inertia, viz., residual char particles, pass through the impaction surface into a wiremesh basket. The flow rate through the center is monitored with a mass-flow transducer, and adjusted during a run to maintain the desired flow split.

Coal tar aerosol is recovered on a four-stage assembly of glass-fiber filters and a polypropylene liner. Under most conditions, $90 \%$ of the aerosol is collected on the filters. Even though the aerosol does not penetrate a single filter element, multiple stages are needed to manage the increasing flow resistance as the aerosol accumulates. The top three stages are punctured with pushpins to decrease their flow resistance as aerosol collects. Even so, most of the aerosol deposits onto these stages. The final stage scavenges the stream at the size threshold of the filters with an efficiency of $99.93 \%$ at 0.3 microns.

Yields of char and aerosol are based on the weight gain of their respective collection elements and the suspension feedrate. All of the solid products from all of the coal fed into the system are recovered. Aerosol yields are adjusted for several factors. An amount is added for the loss of aerosol into the stream that convects char into its basket in the centripeter, based on the flow split inside the centripeter. A fixed amount of 2 daf wt.\% is added to account for losses due to deposition in the quench nozzle, consistent with our previous characterization studies. In addition, our previous aerosol yields included the amount of oils assigned by flame ionization detection. 


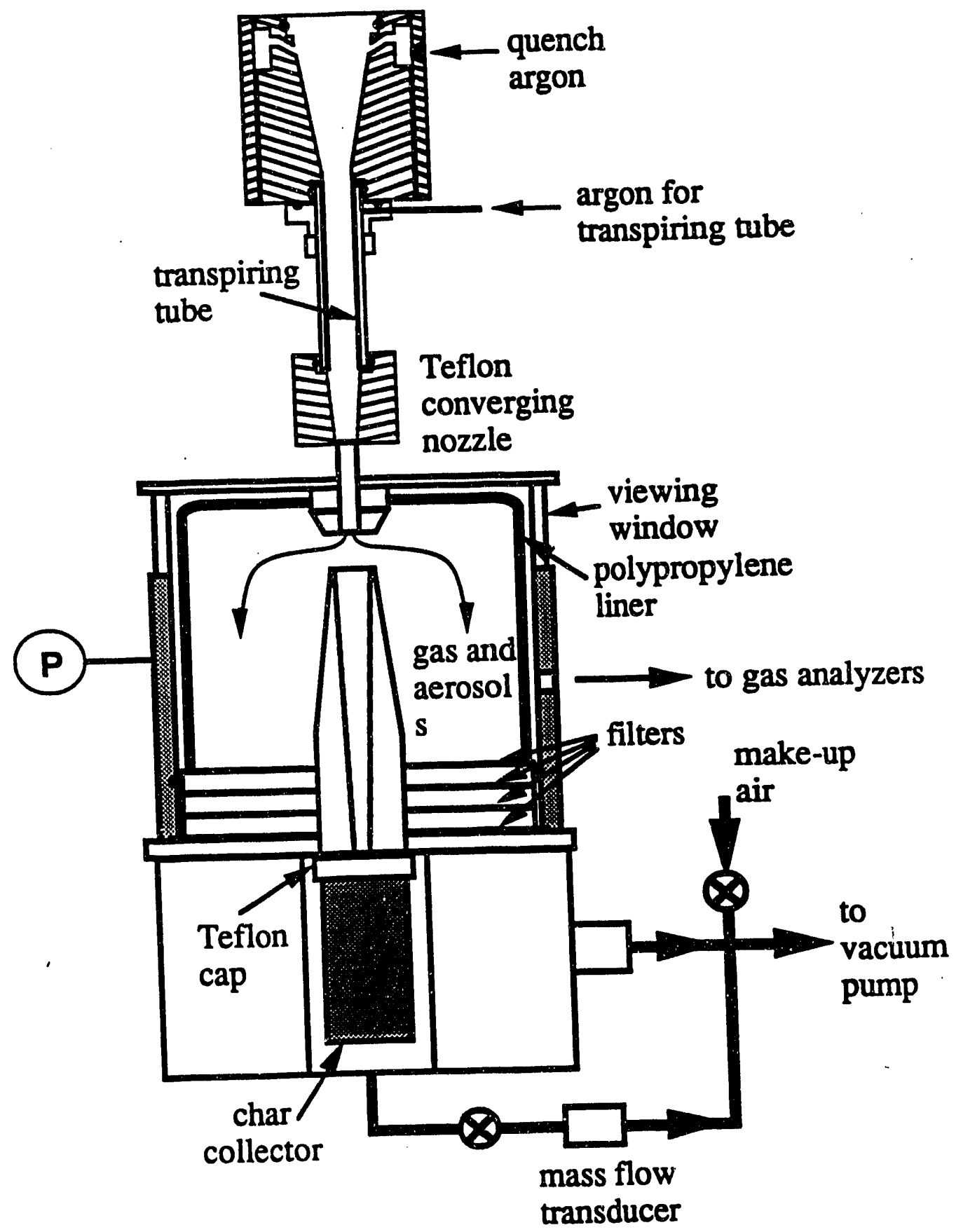

Figure 1. The virtual impactor to recover tar/soot aerosol and char particles. 
We did not perform this analysis in this project, so aerosol yields are assigned somewhat differently here, as the sum of tar and soot. Particularly for high volatile bituminous coals, they will be significantly lower because oils yields can approach $10 \mathrm{wt} . \%$.

Soot is recovered from the aerosol by extraction and filtration. Aerosols on the glass-fiber filters in the centripeter are first extracted into THF in an ultrasonic bath. Then the solution is passed through a Teflon filter with 0.2 -micron pores. The dry weight of the membrane residue is denoted as the soot yield. Tar yields are assigned as the difference between the aerosol and soot yields, once all of the corrections discussed earlier have been applied. The PAH solutions are stored in amber glass vials under nitrogen in a freezer for subsequent analyses.

In the preliminary studies to identify the best furnace temperature for primary devolatilization studies, weight loss and tar yields were monitored at various temperatures. The weight fraction of soot in the aerosol product was evaluated from the extraction and filtration step just described. The best temperature was chosen on the basis of the highest total weight loss and the lowest soot fraction. Maximum weight loss is attained for the highest extent of primary. devolatilization. In fact, there is an ultimate yield for primary devolatilization that depends on coal rank and heating rate, among other factors. In our previous studies in the short furnace, asymptotic weight loss values came within a few weight percentage points of the true ultimate values determined under more severe reaction conditions for coal types across the rank spectrum, yet the soot fraction in the aerosol was negligible in all cases.

This near-perfect performance was desirable for detailed characterizations of the complete distributions of products throughout primary and secondary pyrolysis that we have already published. But the tolerances are not nearly as strict for PAH preparation in this project. It is essential that we know the extents of primary devolatilization (and secondary pyrolysis) for all of our PAH samples, but it is much less important to know that samples are available for complete 
primary devolatilization and absolutely no secondary pyrolysis. As long as a broad range of values for extents of primary devolatilization (and secondary pyrolysis) are represented, the objectives of this project are being met.

\subsubsection{Experimental Results}

Many preliminary runs were made this quarter to identify the best temperature for primary devolatilization studies. Most are not worth considering in detail; suffice to show only the best dataset for a furnace temperature of $1380 \mathrm{~K}$. These experiments were run with the Pit. \#8 hvA bituminous sample that we obtained from PETC for previous studies. Its label is PSOC-1451D and its vltimate analysis is 82.5 daf wt.\% $\mathrm{C} ; 5.6 \% \mathrm{H} ; 1.8 \% \mathrm{~N} ; 1.6 \% \mathrm{~S}$; and $8.5 \% \mathrm{O}$ (by diff.). On a dry basis it has $12.6 \%$ ash.

The data covering transient primary devolatilization for the Pit. \#8 appear in Fig. 2. Although we have an optical diagnostic for particle residence time in the hot zone, we have not yet measured particle residence times for the new operating conditions. So the conversion data are plotted versus velocity of the entrainment gas at the furnace inlet, which is inversely proportional to residence time. Notwithstanding this complication, this dataset does establish the extent of primary devolatilization, in terms of values of fractional ultimate yields. The observed values of weight loss span a range from 5 to 49 wt. \%. The lowest value occurred with the highest gas velocity, hence the lowest residence time. This operating condition nearly attains the onset of primary devolatilization. It is not particularly useful for sample preparation, though, because too little PAH is expelled. But it is reassuring that, at very short residence times, the observed yields are indistinguishable from zero. 
วริยานววมว $700 \mathrm{~S}$

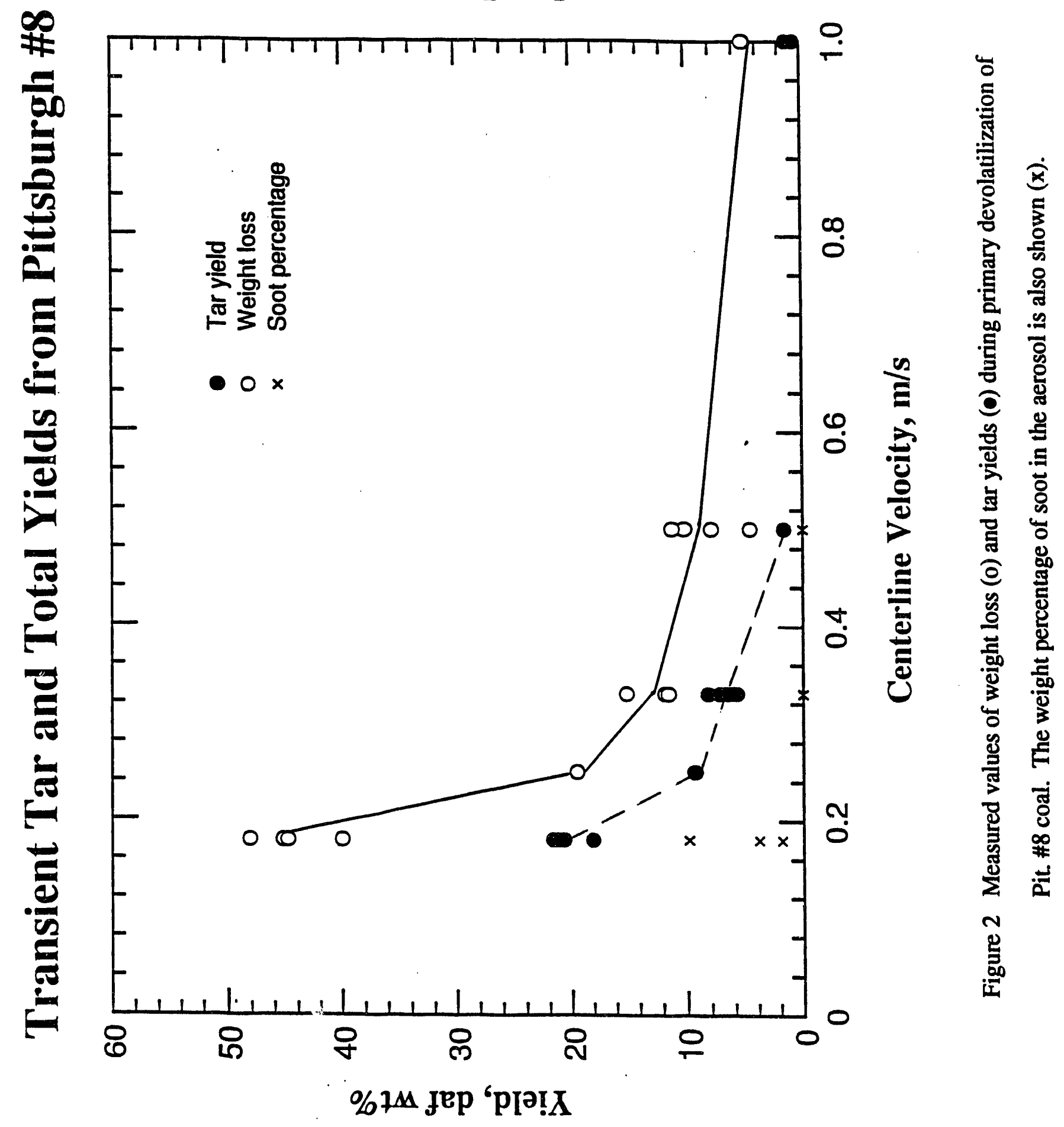


At lower gas velocities, residence times become long enough for much more substantial yields. Weight loss increases continuously while appreciable amounts of PAH are recovered. At the lowest gas velocity of $0.18 \mathrm{~m} / \mathrm{s}$, the tar yield reaches $22 \mathrm{wt} \%$. At first glance, this value seems much lower than our former value for ultimate tar yields from the same Pit. \#8 coal of 31 wt. \%. However, the former ultimate value includes the oils yield, which is $8 \mathrm{wt}$. \%. Clearly these values are within an experimental uncertainty of $2 \mathrm{wt}$ \%.

However, the highest weight loss values observed at $0.18 \mathrm{~m} / \mathrm{s}$ are below the ultimate yields determined previously with a furnace at $1840 \mathrm{~K}$ in the $5 \mathrm{~cm}$ hot zone. The nominal value from the present dataset is $47 \mathrm{wt} . \%$, which is significantly below the former value of $61 \mathrm{wt} . \%$. All products are automatically accounted for in the weight loss data because weight loss is assigned as the difference between the weights of all coal fed and of all char recovered, so the difference is not due to incomplete product recovery and analysis. It is an accurate measure of the impact of the different operating conditions we have used for this study. In fact, the observation that the ultimate tar yield has been achieved before the ultimate total yield is consistent with our previous dataset. That data shows that tar evolution ceases at a weight loss of $46 \mathrm{wt} . \%$, consistent with the newly observed asymptotic tar yield of $22 \mathrm{wt} . \%$ at a weight loss of $47 \mathrm{wt} . \%$. Most important, even though this set of operating conditions does not cover all of primary devolatilization, it does represent the range of extents of devolatilization from 0.08 to 0.77 (on a scale from 0 to 1 ). Moreover, PAH samples represent the entire extent of tar evolution during primary devolatilization.

Of course, higher weight loss would be observed for furnace temperatures hotter than 1380 $\mathrm{K}$. The reason that we did not increase the furnace temperature is apparent in the soot fractions in Fig. 2. There is no soot whatsoever in the PAH recovered at gas velocities higher than $0.25 \mathrm{~m} / \mathrm{s}$. But the ultimate aerosol sample for a velocity of $0.18 \mathrm{~m} / \mathrm{s}$ does show the first indications of secondary pyrolysis. Its soot fraction is only about $5 \%$, so this PAH is representative of primary devolatilization products. But raising the furnace temperature would only increase the soot 
fraction. We therefore chose a furnace temperature of $1380 \mathrm{~K}$ as the best compromise value for all subsequent primary devolatilization experiments.

\subsection{Sample Analysis}

\subsubsection{Acquisition of Standards}

As mentioned in our previous quarterly reports, the coal tar samples being generated are expected to consist of an extremely complex mixture of organic compounds, ranging widely in both molecular weights and polarities. Because of this, to thoroughly characterize the fractionation achieved by the gravity-flow column chromatographic system, we must utilize a suite of standards spanning the range (in both polarities and molecular weights) of organic substances expected to be found in coal tar.

In selecting the standard PAH compounds, consideration was given to including a wide range of ring numbers in order to obtain compounds with different molecular weights. In addition, efforts were made to locate singly-and doubly-substituted PAH standards as well as those with no substituents. Finally, different types of substituents on the PAH were sought in order to better investigate the influence of substituent type on the elution of organics through the prefractionation column.

The seventeen polycyclic aromatic compounds (PAC) selected and obtained are shown in Figure 3. They range in size from naphthalene, with two rings and a molecular weight of 128 , to decacyclane, with 10 rings and a molecular weight of 450 . Five substituted naphthalene compounds were procured, with cyano-, nitro- and carboxy-groups as mono-substituents, as well as dinitro- and dihydroxy-substituted species. PACs with fused nitrogen, oxygen and sulfur in the ring structure were also obtained, as well as an ammonia-substituted PAH. 


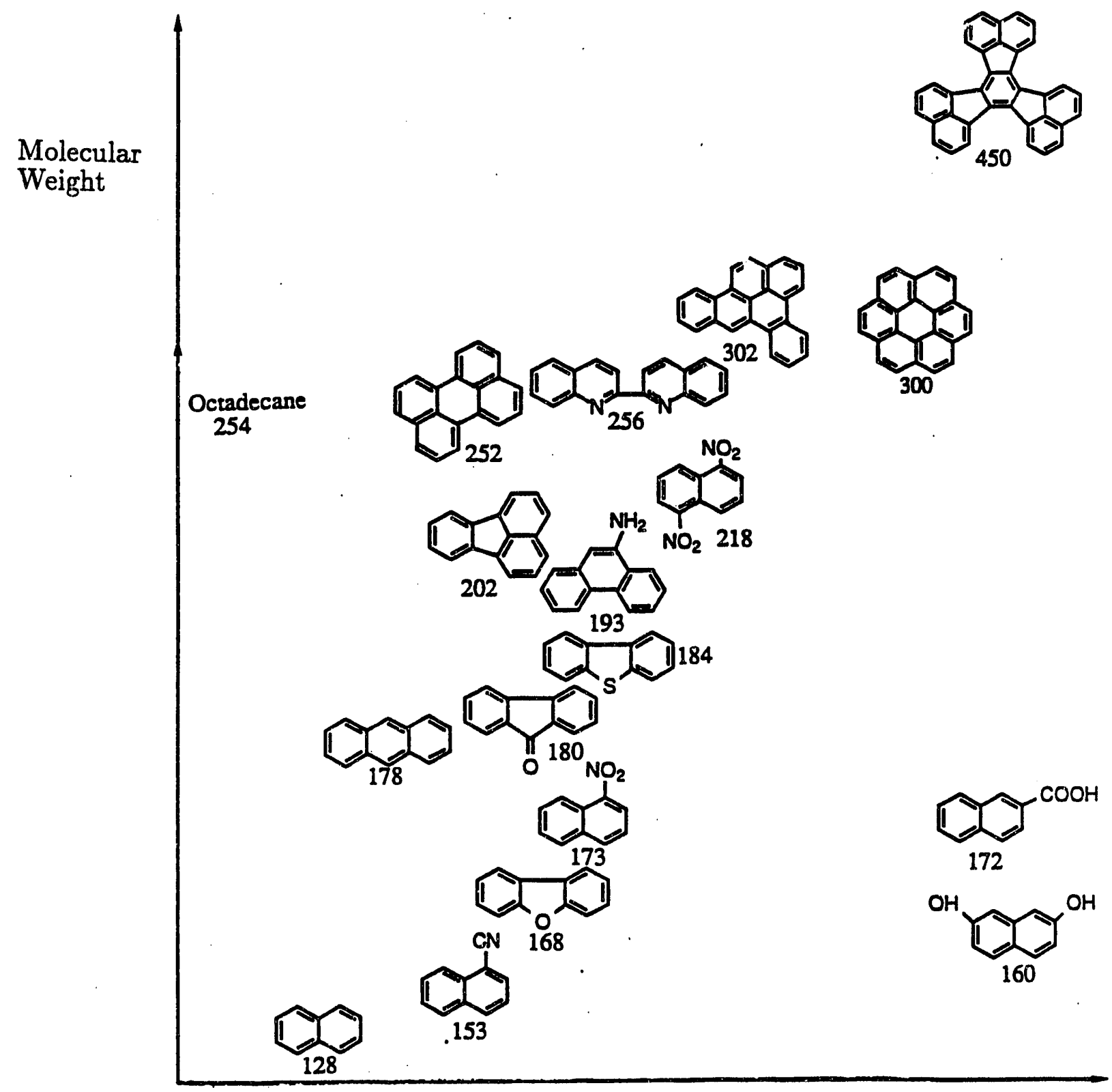

Increasing Polarity of Eluting Solvent

Figure 3. Range of Organic Standards Acquired for Calibration of Fractionation Procedures 
The $x$-axis in Figure 3 represents the expected fractionation of this suite of standards in our gravity-flow fractionation column. It is apparent that both molecular weight and polarity play a role in determining in which fraction a particular compound elutes. Nonetheless, this prefractionation procedure will allow us to separate a wide range of nonpolar PAC from the highly polar compounds prior to analyzing the ring size distribution on the relatively nonpolar fraction via HPLC. In addition, the fraction of each coal tar sample eluting in each solvent may provide additional information regarding the nature of the coal tar as a function of the combustion conditions.

\subsubsection{Prefractionation Results}

Once the assembly of the gravity-flow column chromatographic system was completed, we began testing the prefractionation procedure. using the standard compounds acquired. The main goal of these experiments was to identify what volume of each solvent to sequentially pass through the column in order to achieve: (i) good sample fractionation, and (ii) high sample recovery. While further minor adjustments of these solvent volumes might subsequently prove necessary when fractionating actual coal tar samples, these experiments using standards were expected to give a fairly accurate idea of what approximate solvent volumes would work well.

Octadecane (no rings), naphthalene ( 2 rings), anthracene ( 3 rings), and coronene ( 7 rings) were selected as the standards to be used for this initial calibration of the prefractionation procedure. After loading the sample of standard onto the column, several small aliquots (0.5-1 ml) of each solvent were sequentially added, and the eluant from each aliquot was collected in a separate vial. Quantification of the mass of standard recovered sequeintially in each vial provided an indication of what total volume of each solvent was most appropriate for attaining good fractionation and recovery. 
A recovery curve obtained inr the coronene standard is shown in Figure 4. Each point on the curve represents a separate vial of eluant; the smoothness of the curve from 0 to $19 \mathrm{ml}$ indicates that the volumes of solvent used are the minimum necessary to achieve a good fractionation of the sample. The last aliquot of methanol used (from $19-22 \mathrm{ml}$ ) recovered almost no additional sample, indicating that a total of $4 \mathrm{ml}$ of methanol (rather than $7 \mathrm{ml}$ ) may be adequate.

As expected from previous work, all of the octadecane sample was observed to elute in the heptane (most nonpolar) fraction, while most of the naphthalene and anthracene samples eluted in the toluene step. As shown in Figure 4, over half of the recovered coronene eluted in the more polar dichloromethane fraction, with another one-quarter eluting later in the methanol and the remainder eluting earlier in the toluene.

Sample recoveries varied considerably with the standard compound used. While essentially $100 \%$ recovery was achieved repeatedly for octadecane, regardless of the sample size, $90-97 \%$ of the anthracene standard was recovered, and $67-76 \%$ of the coronene sample (both with better recoveries for the larger-sized samples). However, these values should be considered as preliminary since these experiments were performed while simultaneously testing and modifying various steps in the prefractionation procedure. It is expected that, with further refinements, recoveries of $\geq 85 \%$ will routinely be achieved for all samples. 


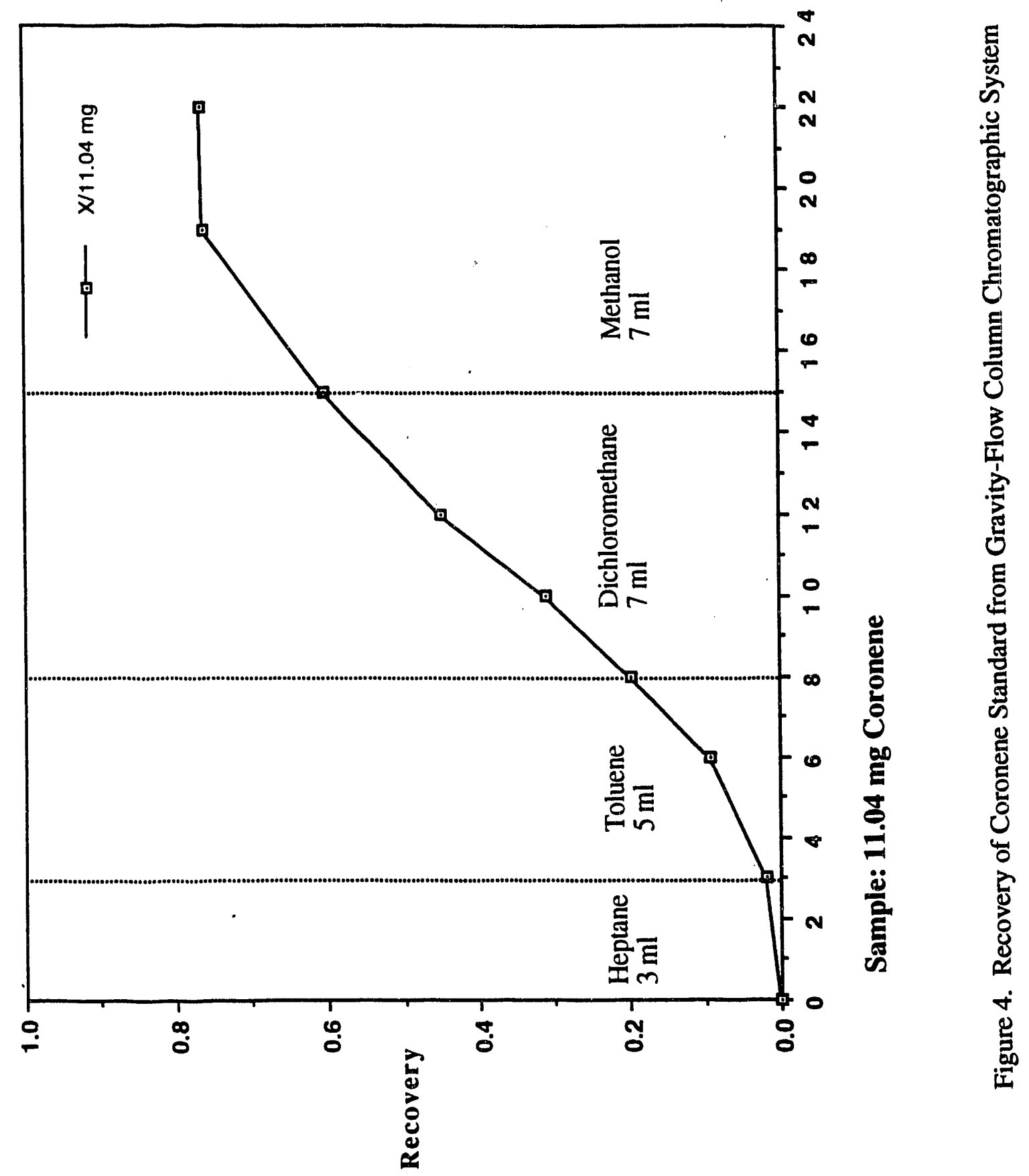




\section{SHORT RANGE OBJECTIVES}

During the coming quarter, Mr. Dadamio will reproduce the results of past secondary pyrolysis studies using identical conditions. Pending this validation of the experimental technique, he will move on to generate PAH samples reflecting controlled extents of primary devolatilzation and secondary pyrolysis for other coal types.

Mr. Yu's goal for next quarter will be to begin prefractionation of genuine coal tar using samples from combustion of Pittsburgh \#8, a high-volatile bituminous coal. Further examination of the optimal solvent volumes will be conducted, as well as the effect of sample size on the total sample recovery. 

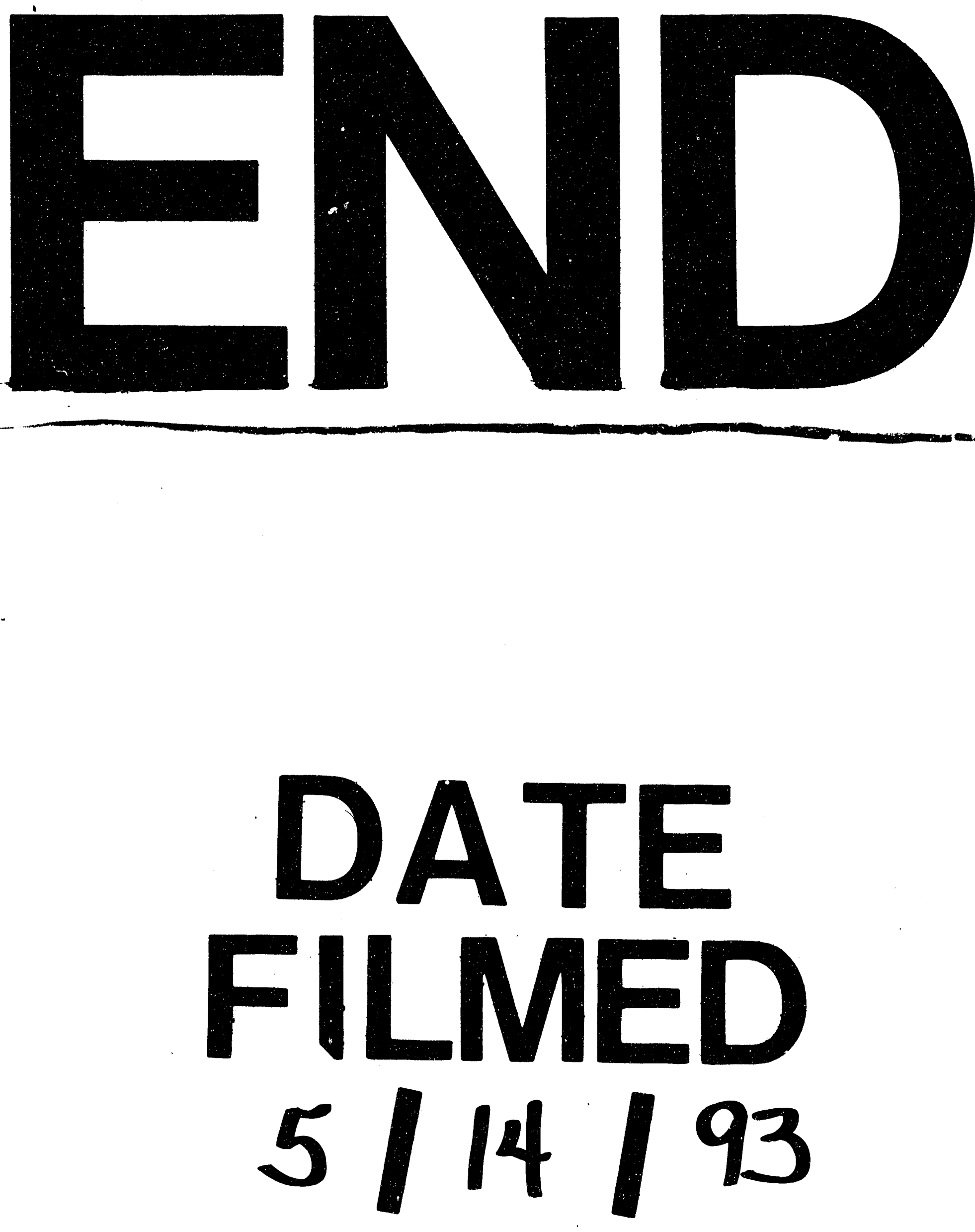
